\title{
miR-106b-5p inhibits the invasion and metastasis of colorectal cancer by targeting CTSA
}

This article was published in the following Dove Press journal:

OncoTargets and Therapy

\author{
Shujuan $\mathrm{Ni}^{1,2, *}$ \\ Weiwei Weng ${ }^{1,2, *}$ \\ Midie $\mathrm{Xu}{ }^{1,2}$ \\ Qifeng Wang ${ }^{1,2}$ \\ Cong Tan ${ }^{1,2}$ \\ Hui Sun ${ }^{1,2}$ \\ Lei Wang ${ }^{1,2}$ \\ Dan Huang ${ }^{1,2}$ \\ Xiang Du ${ }^{1,2}$ \\ Weiqi Sheng ${ }^{1,2}$ \\ 'Department of Pathology, Fudan \\ University Shanghai Cancer Center, \\ Shanghai, China; ${ }^{2}$ Department of \\ Oncology, Shanghai Medical College, \\ Fudan University, Shanghai, China \\ *These authors contributed equally \\ to this work
}

Correspondence: Weiqi Sheng

Fudan University Shanghai Cancer

Center, 270 Dongan Road,

Shanghai 200032, China

Tel +862164175590

Email shengweiqi2006@I63.com

\begin{abstract}
Background: Although miR-106b-5p has been reported to play a pivotal role in various human malignancies, its role in colorectal cancer (CRC) remains unknown. In this study, we comprehensively investigated miR-106b-5p expression and biologic functions in CRC and the molecular mechanism involved.
\end{abstract}

Patients and methods: miR-106b-5p expression was detected in CRC tissues and cell lines by quantitative reverse transcription-polymerase chain reaction. The effects of miR-106b-5p on metastasis were determined in vitro using transwell assays, and in vivo effects were evaluated using a mouse tail vein injection model. Downstream targets of miR-106b-5p were confirmed using bioinformatics programs, luciferase assays, and rescue experiments. Target gene expression and clinicopathologic parameters were also analyzed.

Results: miR-106b-5p expression was lower in CRC tissues than in corresponding nontumorous tissues ( $P=0.009$ ), and miR-106b-5p downregulation was negatively associated with lymph node metastasis $(P=0.006$ ). Functional assays demonstrated that miR-106b-5p overexpression suppressed CRC cell migration and invasion in vitro and lung metastasis formation in vivo. In addition, luciferase assays confirmed that miR-106b-5p directly bound to the $3^{\prime}$ untranslated region of cathepsin A (CTSA) and that miR-106b-5p suppressed CRC cell migration and invasion by targeting CTSA. Clinicopathologic analysis showed that CTSA was significantly upregulated in $\mathrm{CRC}$, and increased CTSA was negatively associated with lymph node metastasis $(P=0.012)$.

Conclusion: Our findings revealed that miR-106b-5p inhibits CRC metastasis by upregulating CTSA expression, which may lead to novel therapeutic strategies for CRC patients.

Keywords: colorectal cancer, metastasis, miR-106b-5p, cathepsin A

\section{Introduction}

Colorectal cancer (CRC) ranks as the third most prevalent cancer worldwide and accounts for $\sim 9 \%$ of all cancer cases. ${ }^{1}$ Despite clinical implementation of numerous therapeutic strategies, CRC remains a leading cause of cancer-related death because of therapy resistance and metastasis. Most CRC patients with distant metastasis are not suitable candidates for surgical treatment and have poor outcomes. ${ }^{2}$ Therefore, understanding the molecular mechanisms and genetic alterations underlying CRC would have major implications on its precise diagnosis and novel treatment strategies.

MicroRNAs (miRNAs) are a class of small, endogenous noncoding RNAs that play important roles in the regulation of target genes by complementary pairing with the $3^{\prime}$ untranslated regions ( $3^{\prime}$ UTRs) of mRNAs, leading to translational repression or mRNA degradation. ${ }^{3}$ Evidence has shown that miRNAs are abnormally expressed in various cancers, and dysregulated miRNAs are associated with tumor initiation, promotion, and progression. ${ }^{4-9}$ 
Over the past decade, several studies have also reported that miRNAs affect the expression of genes and pathways involved in CRC pathogenesis, ranging from initiation to metastasis. ${ }^{10-15}$ In a previous study, we identified the expression profiles of miRNAs in CRC using microarrays, and the results functionally implicated several miRNAs in carcinogenesis and progression. ${ }^{5,16}$ Among them, miR-106$\mathrm{b}-5 \mathrm{p}$ is the most upregulated miRNA in cancer tissue. miR106b-5p expression is reportedly elevated in non-small-cell lung carcinoma, ${ }^{17}$ hepatocellular carcinoma, ${ }^{18}$ and clear cell renal cell carcinoma, ${ }^{19}$ thus exerting an oncogenic effect on these cancer types. However, whether reduced expression of miR-106b-5p is involved in CRC initiation and development has not yet been clarified.

In the present study, we verified that miR-106b-5p suppresses CRC metastasis by targeting cathepsin A (CTSA), results that offer a probable novel strategy for the treatment of CRC metastasis.

\section{Patients and methods}

\section{Patients and samples}

Human CRC tissues and their corresponding nontumor tissues were collected from 78 patients with $\mathrm{CRC}$ at the time of surgical resection from 2005 to 2008 at the Department of CRC at Fudan University Shanghai Cancer Center (FUSCC). All frozen samples were obtained from the tissue bank of FUSCC. Signed informed consent was obtained from all patients, and the study was approved by the clinical research ethics committee of FUSCC. CRC diagnoses were histopathologically confirmed by two pathologists. None of the patients underwent preoperative treatment.

\section{Cell culture}

The HEK-293T cell line and eight human CRC cell lines, HCT116, LoVo, DLD1, HCT8, HT29, SW480, SW620, and $\mathrm{CaCo}$, were purchased from American Type Culture Collection. HEK-293T cells were cultured in DMEM (Gibco, Carlsbad, CA, USA) supplemented with $10 \%$ fetal bovine serum (FBS; Gibco), $50 \mathrm{U} / \mathrm{mL}$ penicillin, and $50 \mu \mathrm{g} / \mathrm{mL}$ streptomycin (Gibco). The human CRC cell lines HCT116, LoVo, DLD1, HCT8, HT29, SW480, SW620, and CaCo2 were cultured in RMPI-1640 (Gibco) supplemented with 10\% FBS (Gibco), $50 \mathrm{U} / \mathrm{mL}$ penicillin, and $50 \mu \mathrm{g} / \mathrm{mL}$ streptomycin (Gibco). All cell lines were maintained at $37^{\circ} \mathrm{C}$ and $5 \%$ $\mathrm{CO}_{2}$ in a humidified atmosphere.

\section{Plasmid construction and cell transfection}

The pri-miR-106b sequence was amplified from normal human genomic DNA and constructed into the lentivirus expression vector pGIPZ to generate pre-miR-106b. For luciferase reporter experiments, $3^{\prime}$ UTR segments containing miR-106b-5p CTSA binding sites were amplified from human genomic DNA by polymerase chain reaction (PCR) and inserted into the psi-CHECK ${ }^{\mathrm{TM}}-2$ vector (Promega, Madison, WI, USA) immediately downstream of the luciferase stop codon. DNA segments with scrambled target sites (CTSA-mutation [MUT]) designed to interfere with seed sequence recognition were also cloned to serve as controls for specificity. All constructs were verified by sequencing.

\section{Lentivirus production and transduction}

Virus particles were harvested 48 hours after cotransfecting pGIPZ-miR-106b and pLVX-CTSA with the packaging plasmid ps-PAX2 and the envelope plasmid pMD2G into HEK293T cells using Lipofectamine 3000 Reagent (Invitrogen, Carlsbad, CA, USA). HCT116 and LoVo cells were infected with recombinant lentivirus-transducing units plus $6 \mathrm{mg} / \mathrm{mL}$ polybrene (Sigma, St Louis, MO, USA).

Oligonucleotide transfection miR-106b-5p inhibitors, miR-106b-3p, miR-106b-mimics, miR-106b-3p mimics, and siRNA against CTSA were synthesized by Ribobio (Guangzhou, China). Oligonucleotide transfection was performed using Lipofectamine 3000 Reagent according to the manufacturer's protocol. The miR-106b-5p inhibitors, miR-106b-3p, miR-106b-mimics, and miR-106b-3p mimics in the transfection system were used at a final concentration of $50 \mathrm{nM}$.

\section{Luciferase reporter assay}

Wild-type and mutated miR-106b-5p putative targets on the CTSA 3' UTR were cloned into the psi-CHECK ${ }^{\mathrm{TM}}-2$ vector. HEK-293T cells were cultured in 96-well plates and cotransfected with 20 pmol of the miR-106b-5p mimic (or NC mimic), $50 \mathrm{ng}$ of luciferase reporters, and $50 \mathrm{ng}$ of a control luciferase plasmid using Lipofectamine 3000 . After 48 hours of transfection, firefly and Renilla luciferase activities were measured using the dual-luciferase reporter assay system (Promega, Madison, WI, USA) according to the manufacturer's protocol. Three independent experiments were performed, and the data are presented as the mean \pm SD.

\section{Cell migration and invasion assays}

Cell migration and invasion ability was measured using 24-well transwell plates ( $8 \mu \mathrm{m}$ pore size; Corning, New York, USA). Briefly, $10 \times 10^{4}$ cells in $200 \mu \mathrm{L}$ of serum-free medium were plated in the top chamber lined with a noncoated membrane, and serum-supplemented medium was used as a 
chemoattractant in the lower chamber. For invasion assays, chamber inserts were coated with $200 \mathrm{mg} / \mathrm{mL}$ matrigel before the cells were plated. After incubation at $37^{\circ} \mathrm{C}$ for 8 or 24 hours, the invading cells on the underside of the membrane were fixed in $100 \%$ methanol stained with crystal violet and counted under a microscope. The mean of triplicate assays for each experimental condition was used.

\section{In vivo tumor metastasis assays}

For in vivo metastasis assays, LoVo cells infected with either the miR-106b-overexpressing lentivirus or the mock lentivirus were transplanted into nude mice (5-week-old $\mathrm{BALB} / \mathrm{c}-\mathrm{nu} / \mathrm{nu}, 6$ per group, $1.5 \times 10^{6}$ cells for each mouse) through the lateral tail vein. After 7 weeks, the mice were killed. Their lungs were removed and subjected to H\&E staining. All research involving animals complied with protocols approved by the Shanghai Medical Experimental Animal Care Commission and was carried out according to the UK Animals (Scientific Procedures) Act.

\section{RNA extraction and quantification and quantitative reverse transcription (qRT)-PCR}

Total RNA was extracted using TRIzol reagent (Invitrogen), and cDNA was synthesized with the PrimeScript RT reagent kit (Takara Bio Inc., Dalian, China). qRT-PCR analyses were carried out to detect mRNA expression using SYBR Premix Ex Taq (Takara Bio Inc.), and glyceraldehyde 3-phosphate dehydrogenase (GAPDH) was used as an internal control. The following primers were used: CTSA forward 5'-ACAAAGACCTGGAATGCG-3' and reverse 5'-CCAAATCCTGGACCACAAC-3'; GAPDH forward 5'-CCGGGAAACTGTGGCGTGATGG-3' and reverse 5' -AGGTGGAGGAGTGGGTGTCGCTGTT-3'.

TaqMan miRNA assays (Applied Biosystems, Foster City, CA, USA) were used to quantitate the expression levels of mature miR-106b-5p and miR-106b-3p according to the manufacturer's instructions. U6 small nuclear RNA was used as an internal control. The qRT-PCR results were defined from the threshold cycle $(\mathrm{Ct})$, and relative expression levels were calculated by using the $2^{-\Delta \Delta \mathrm{CT}}$ method.

\section{Western-blot analysis}

According to standard Western-blot procedures, proteins were separated by $8 \%$ sodium dodecyl sulfate-polyacrylamide gel electrophoresis and then transferred to nitrocellulose membranes (Bio-Rad, Hercules CA, USA). After blocking in $5 \%$ nonfat milk, the membranes were incubated with the following primary antibodies: mouse anti-CTSA monoclonal antibody (mAb, 1:2000; Sigma-Aldrich) and rabbit anti$\beta$-actin mAb (1:3,000; Sigma-Aldrich). The proteins were visualized with enhanced chemiluminescence reagents (Pierce, Rockford, IL, USA).

\section{Immunohistochemistry}

Immunohistochemical staining was performed on $5 \mathrm{~mm} \mathrm{sec-}$ tions of paraffin-embedded human CRC tissues and matched nontumor tissues as well as on 49 pairs of CRC and relative liver metastases to determine the expression of CTSA. Briefly, the slides were incubated in a CTSA antibody (1:100; Sigma-Aldrich) overnight. Subsequent steps were performed using a Universal Dako labelled streptavidin biotin + kit and horseradish peroxidase (DAKO, Carpinteria, CA, USA) according to the manufacturer's instructions. Scoring was measured by the cell cytoplasm staining patterns of tumor and nontumor tissues as follows: 0) absent cell cytoplasm staining; 1) weak cell cytoplasm staining; 2) moderate cell cytoplasm staining; and 3) strong cell cytoplasm staining.

\section{Statistical analysis}

Each experiment was repeated three times, and the data are presented as the mean \pm SD (indicated by error bars). The significance of differences between groups was estimated using the chi-squared test, Student's $t$-test, Mann-Whitney U test, Wilcoxon test, or Kruskal-Wallis one-way analysis of variance. Differences between paired groups were analyzed using paired $t$-tests and Wilcoxon matched-pairs signed rank tests. Relationships between miRNA expressions levels and CTSA expression were explored by the Spearman's correlation. $P$-values $<0.05$ were considered statistically significant. SPSS 20.0 package (IBM, Chicago, IL, USA) and GraphPad Prism 6.0 software (GraphPad Software, Inc., La Jolla, CA, USA) were used for statistical analyses and scientific graphing, respectively.

\section{Results Decreased miR-106b-5p in CRC tissue is associated with disease progression and metastasis}

To determine whether miR-106b-5p expression is associated with CRC, we examined miR-106b-5p expression in a cohort consisting of 78 primary CRC tissues relative to that in their pair-matched, adjacent nontumor tissues using qRT-PCR. miR-106b-5p expression was suppressed in CRC tissues compared to that in their corresponding nontumor tissues ( $P=0.009$, Figure 1A). When $78 \mathrm{CRC}$ samples were stratified based on lymph node metastasis status, miR-106b-5p 

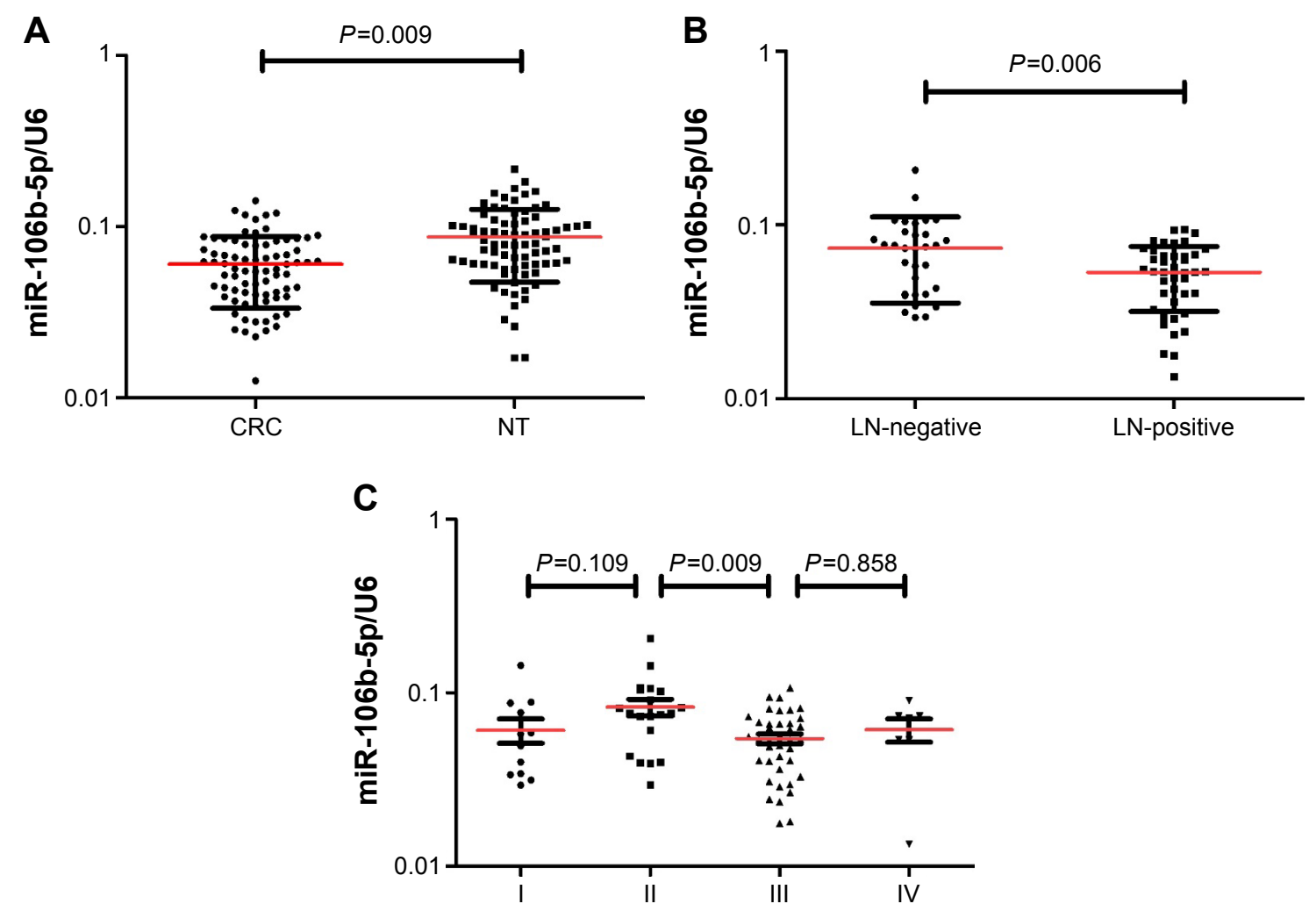

Figure I miR-106b-5p is downregulated in CRC and associated with lymph node metastasis.

Notes: (A) Expression of mature miR-106b-5p in 78 human CRCs and their corresponding NT tissues was determined by TaqMan real-time PCR and normalized to an endogenous U6 RNA control. (B) Downregulation of miR-I06b-5p in CRC was associated with LN metastasis. (C) miR-I06b-5p expression in different clinical stages of CRC. Patients were staged in accordance with the 8th edition of AJCC tumor-node-metastasis classification.

Abbreviations: AJCC, American Joint Committee on Cancer; CRC, colorectal cancer; LN, lymph node; NT, nontumor.

expression was further significantly downregulated in CRC with lymph node metastasis $(P=0.006$; Figure 1B). CRC patients at advanced stages (stages III and IV) showed lower miR-106b-5p expression than patients at early stages (stages I and II; Table 1, Figure 1C). Collectively, the above findings suggest that decreased miR-106b-5p may play an important role in CRC metastasis.

\section{Overexpression of miR-106b-5p suppresses $C R C$ cell migration in vitro and in vivo}

As loss of miR-106b-5p was closely associated with lymph node metastasis in human CRCs, we hypothesized that miR-106b-5p may be involved in the migratory and invasive abilities of CRC cells. Thus, we established LoVo and HCT116 cell lines, CRC cell lines with low basal levels of miR-106b-5p and miR-106b-3p, stably expressing miR-106b by lentivirus infection (Figure 2A).

Intriguingly, overexpression of miR-106b-5p significantly suppressed the migratory and invasive abilities of CRC cells (Figure 2B). Conversely, we transiently transfected
Table I The relationship between miR-106b-5p expression and clinicopathologic parameters in primary CRCs

\begin{tabular}{|c|c|c|c|}
\hline Characteristics & CRC $(n=78)$ & $\begin{array}{l}\text { Median expression } \\
\text { of miR-106b-5p }\end{array}$ & $P$-value* \\
\hline \multicolumn{4}{|l|}{ Gender } \\
\hline Male & 45 & $0.059|8 \pm 0.004| 28$ & 0.187 \\
\hline Female & 33 & $0.06883 \pm 0.0063 \mathrm{II}$ & \\
\hline \multicolumn{4}{|l|}{ Age at diagnosis } \\
\hline$>6$ & 35 & $0.06523 \pm 0.005606$ & 0.548 \\
\hline$\leq 60$ & 43 & $0.06085 \pm 0.004 \mid 45$ & \\
\hline \multicolumn{4}{|l|}{ Nodal status } \\
\hline Positive & 45 & $0.05347 \pm 0.003345$ & 0.006 \\
\hline Negative & 33 & $0.07334 \pm 0.006684$ & \\
\hline \multicolumn{4}{|l|}{ TNM stage s $^{\ddagger}$} \\
\hline $\mathrm{I}+\mathrm{II}$ & 33 & $0.07334 \pm 0.006684$ & 0.006 \\
\hline III+IV & 45 & $0.05347 \pm 0.003345$ & \\
\hline \multicolumn{4}{|l|}{ Local invasion } \\
\hline $\mathrm{TI}+\mathrm{T} 2$ & 16 & $0.06008 \pm 0.007376$ & 0.656 \\
\hline $\mathrm{T} 3+\mathrm{T} 4$ & 62 & $0.06409 \pm 0.004 \mid 24$ & \\
\hline \multicolumn{4}{|l|}{ Tumor location } \\
\hline Rectum & 36 & $0.06506 \pm 0.005363$ & 0.592 \\
\hline Colon & 42 & $0.06117 \pm 0.004693$ & \\
\hline
\end{tabular}

Notes: *The Mann-Whitney $U$ test; ${ }^{\text {*}}$ patients were staged in accordance with the 8th edition of the AJCC TNM classification.

Abbreviations: AJCC, American Joint Committee on Cancer; CRC, colorectal cancer; TNM, tumor-node-metastasis. 
A

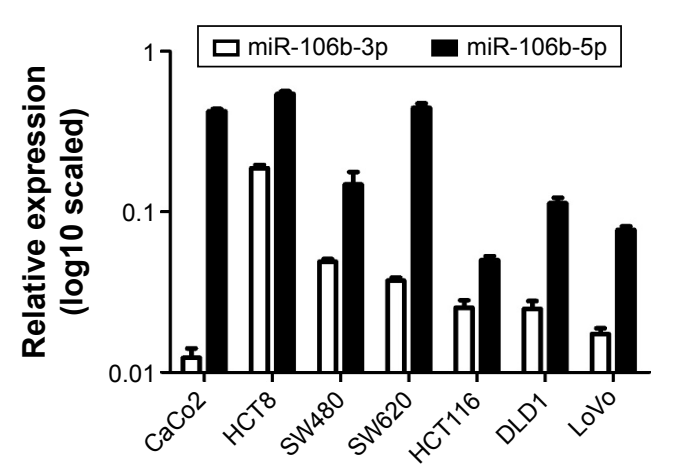

D

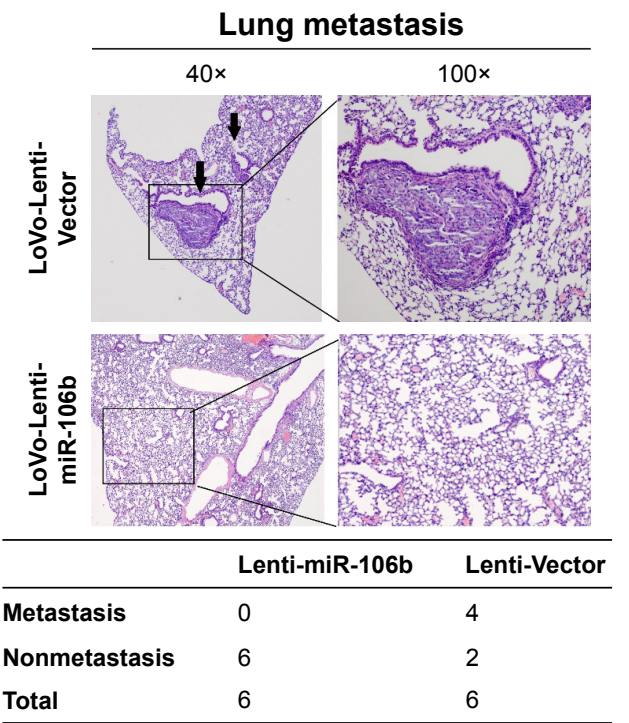

B
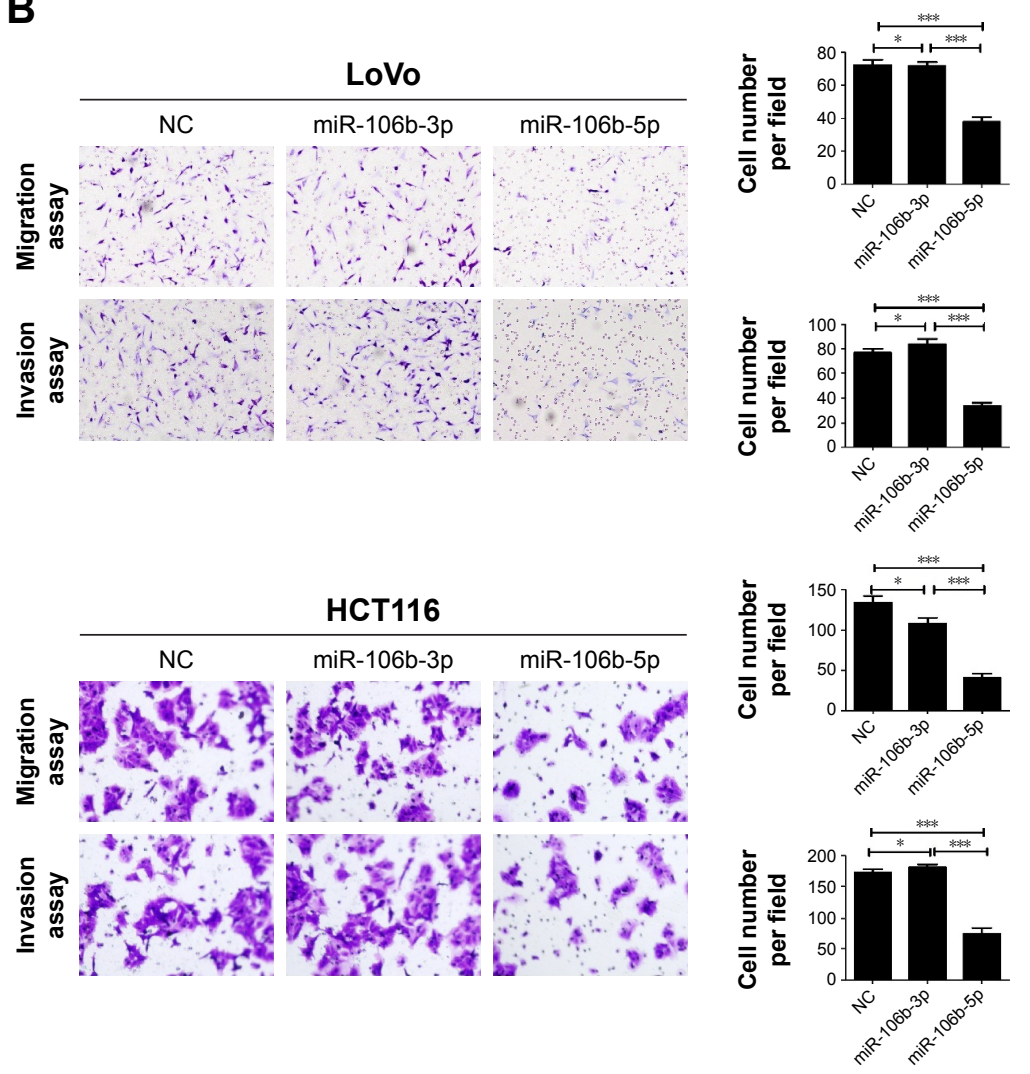

C

НСТ8

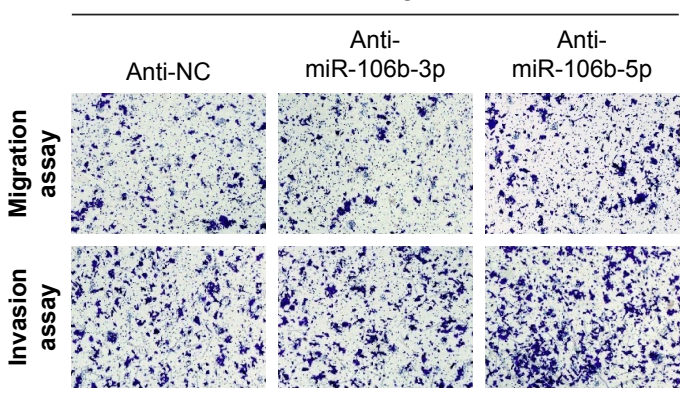

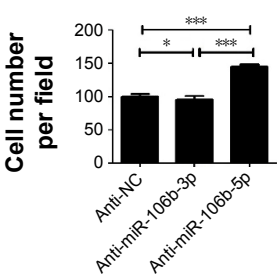

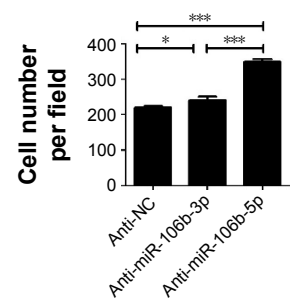

Figure 2 miR-106b-5p suppresses CRC cell invasion in vitro and metastasis in vivo.

Notes: (A) Expression levels of miR-106b-5p and miR-106b-3p were determined by TaqMan real-time PCR in CRC cell lines. U6 RNA served as an internal control. (B) Transwell migration and invasion assays in LoVo and HCTII6 cells transfected with an miR-I06b-3p mimic, miR-I06b-5p mimic, or NC. (C) Transwell migration and invasion assays in HCT8 cells transfected with miR-106b-3p inhibitors, miR-106b-5p inhibitors, or an NC inhibitor. (D) Representative H\&E-stained sections of lung tissues isolated from mice injected with LoVo-Lenti-Vector or LoVo-Lenti-miR-I06b cells through the tail vein. The arrow head points to the tumor focus formed in the lung $(* P>0.05, * * * P<0.001)$.

Abbreviations: CRC, colorectal cancer; PCR, polymerase chain reaction; NC, negative control.

the miR-106b-5p inhibitor into HCT8 cells, which had a relatively high endogenous miR-106b-5p expression level compared to that in the other CRC cell lines (Figure 2C). Knocking down miR-106b-5p increased CRC cell migration and invasion. Furthermore, miR-106b-3p, which processes from the $3^{\prime}$ end arm of pre-miR-106b, did not significantly affect $\mathrm{CRC}$ cell migration and invasion (Figure 2B and C).
To further explore the role of miR-106b-5p in tumor metastasis in vivo, we transplanted LoVo cells stably expressing miR-106b-5p into nude mice through the tail vein. Histologic analysis of the mouse lungs confirmed that miR106b-5p suppressed lung metastasis formation. The numbers and size of lung metastasis nodules were significantly decreased in the LoVo-miR-106b-5p group compared to 
those in the LoVo vector group (Figure 2D). Taken together, our results suggest that miR-106b-5p is a negative regulator of CRC metastasis.

\section{miR-I06b-5p post-transcriptionally reduces CTSA expression by directly targeting its 3' UTR}

To explore the molecular mechanism of miR-106b-5p in CRC metastasis, we used the TargetScan and Miranda programs to search for putative protein-coding gene targets of miR-106b-5p, and a total of 129 downregulated genes were selected. Because miR-106b-5p is known to inhibit CRC metastasis, its target gene should promote tumor cell invasion and metastasis. Based on this rationale, eight candidate genes (ATAD2, BTG3, CTSA, STAT3, MAP3K5, DDX5,
$E 2 F 5$, and $F G D 4$ ) were selected after a literature review (Figure 3A). We performed qRT-PCR to screen the genes downregulated by miR-106b-5p, revealing that the expression levels of CTSA, ATAD2, BTG3, and FGD4 mRNA were most downregulated by miR-106b-5p in the HTC116 and LoVo cells stably expressing miR-106b-5p (Figure 3B). In addition, Western-blot analysis showed that forced miR-106b-5p expression silenced endogenous CTSA protein expression in LoVo cells, while transfection of the miR-106b-5p inhibitor increased CTSA expression in HCT8 cells; miR-106b-5p had no significant effects on ATAD2, BTG3, and FGD4 protein expression (Figure 3C). These results suggest that CTSA expression is regulated by miR-106b-5p in CRC.

Analysis of the CTSA 3' UTR sequence using TargetScan revealed two possible binding sites for miR-106b-5p,
A

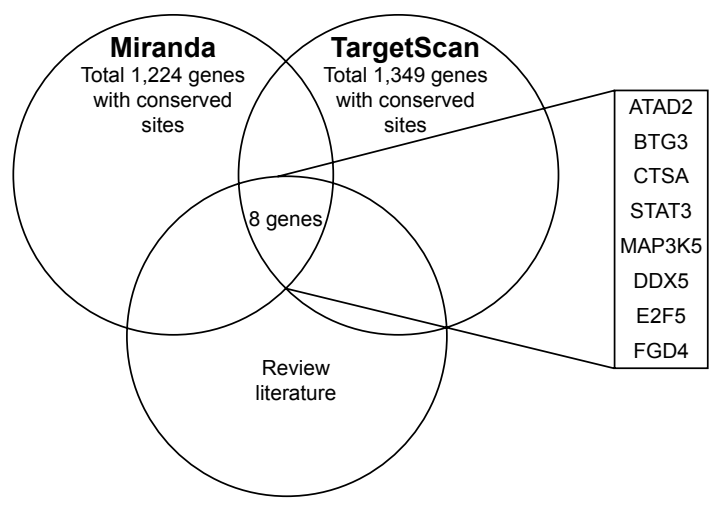

B

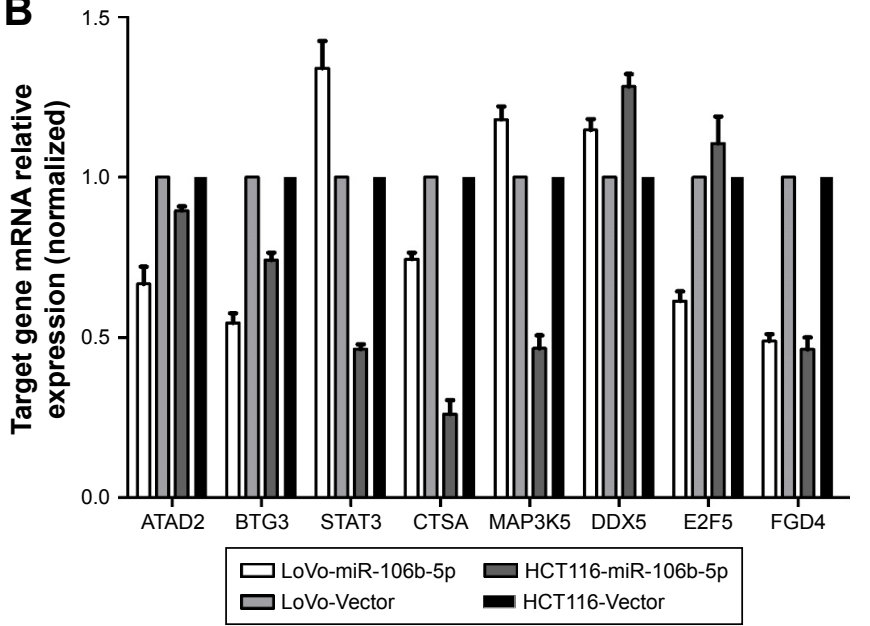

C

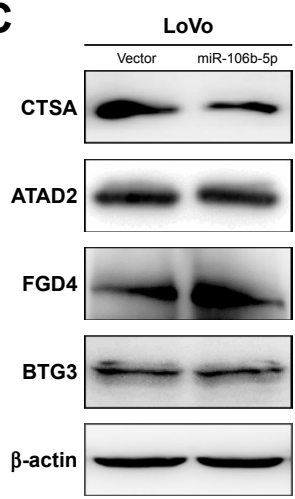

D

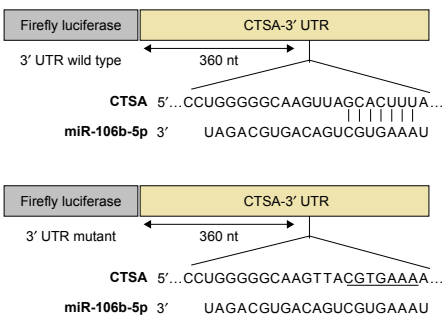

miR-106b-5p $3^{\prime}$ UAGACGUGACAGUCGUGAAAU
E

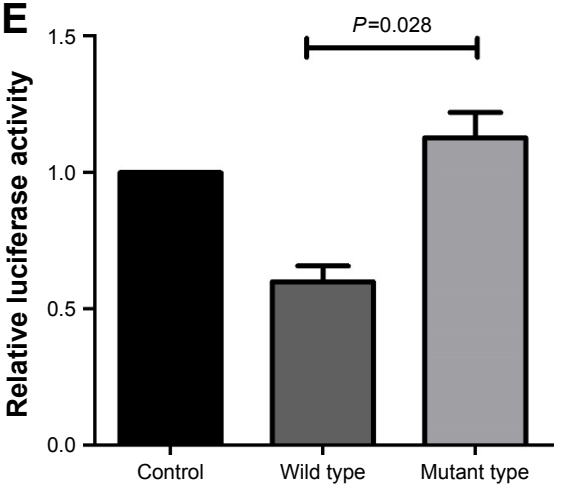

Figure 3 miR-106b-5p negatively regulates CTSA by binding to the CTSA $3^{\prime}$ UTR.

Notes: (A) Initial screening of miR-106b-5p target genes in HCTII6 and LoVo cells using bioinformatics predictions and literature review. A total of eight downregulated genes were selected. (B) The mRNA levels of FGD4, ATAD2, BTG3, and CTSA were determined by qRT-PCR in HCTII6 and LoVo cells stably expressing miR-106b-5p. $\beta$-Actin served as an internal control. (C) Western-blot analysis was used to detect the expression levels of endogenous FGD4, ATAD2, BTG3, and CTSA in LoVo cells infected with an miR-106b-5p-expressing lentivirus or a control lentivirus and in HCT8 cells transfected with an miR-106b-5p inhibitor or an NC inhibitor. $\beta$-Actin served as an internal control. (D) Model of the construction of wild-type and mutant psi-CHECKTM-2-CTSA-3' UTR vectors. The wild-type and mutant (underlined) miR-I06b-5p binding sites on the CTSA 3' UTR are shown. (E) Luciferase activity assays of luciferase vectors with wild-type or mutant CSTA 3' UTRs were performed after cotransfection with miR-106b-5p mimics or an NC mimic. Luciferase activity was normalized to that of Renilla luciferase. The normalized luciferase activity of the vector and NC transfection was set as relative luciferase.

Abbreviations: CTSA, cathepsin A; UTR, untranslated region; NC, negative control. 
indicating that the CTSA gene transcript may be a direct target of miR-106b-5p. Thus, we directly fused a series of CTSA 3' UTR fragments, including the full-length construct, binding site 1 , binding site 2 , and their corresponding mutant counterparts, downstream of the firefly luciferase gene (psi-CHECK ${ }^{\mathrm{TM}}-2$; Figure 3D and E). miR-106b-5p decreased the relative luciferase activity of the full-lengthCTSA 3' UTR construct. In contrast, luciferase activity of the counterpart with both sites mutated was not significantly altered, indicating that such regulation was dependent on specific sequences. Taken together, these results indicate that miR-106b-5p downregulates CTSA expression by directly targeting its $3^{\prime}$ UTR.

\section{miR-106b-5p suppresses CRC cell} migration and invasion by targeting CTSA

CTSA is closely associated with tumor invasion and metastasis. ${ }^{20}$ However, the role of CTSA in the miR-106b-5pmediated effects on CRC has not been characterized. To determine whether the dysregulation of CTSA is involved in the regulation of cell migration and invasion by miR-106b-5p, we used specific siRNAs against CTSA to knock down CTSA expression (Figure 4A) and confirmed that expression of the CTSA protein was suppressed by miR-106b-5p in CRC cells (Figure 4B). Transwell assays showed that CTSA suppression partially recovered the effects of miR-106b-5p
A
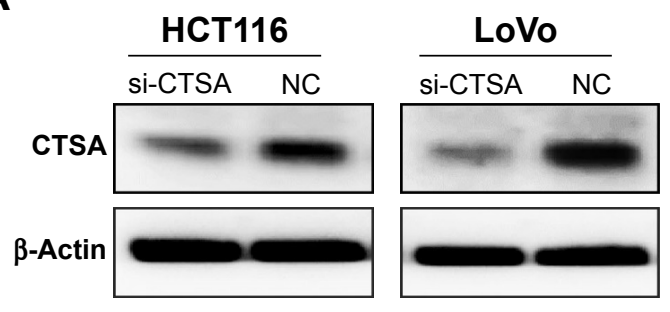

C
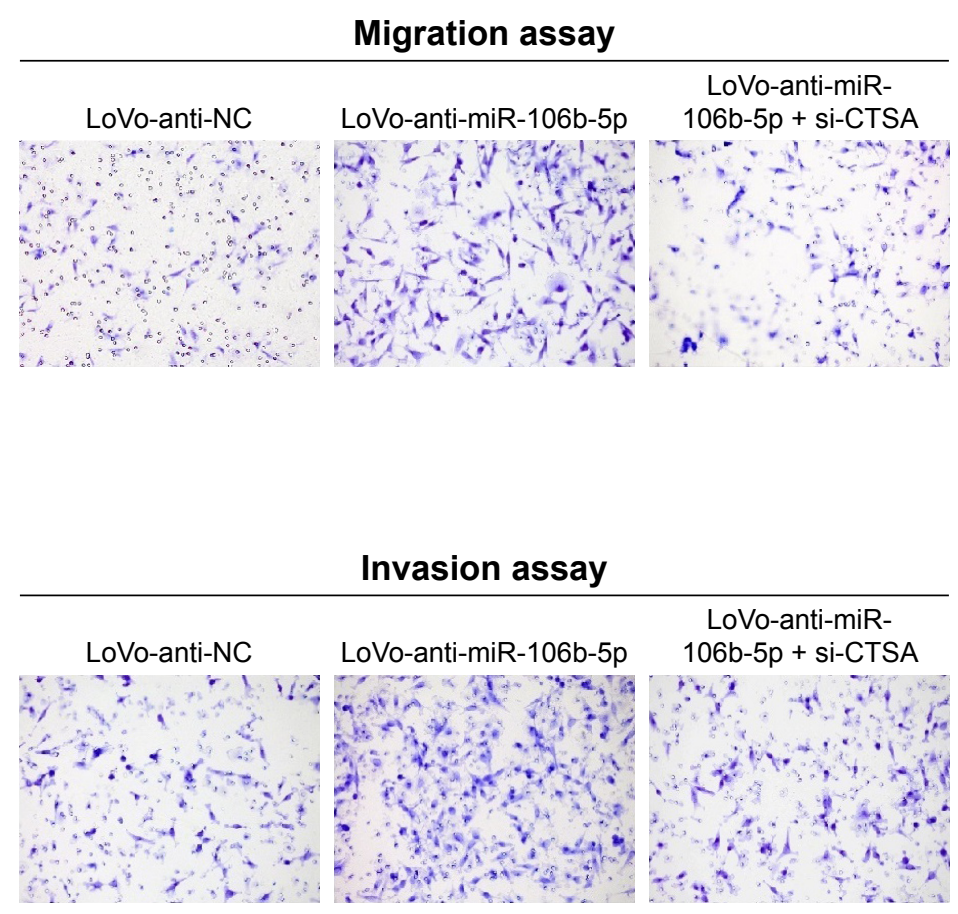

B
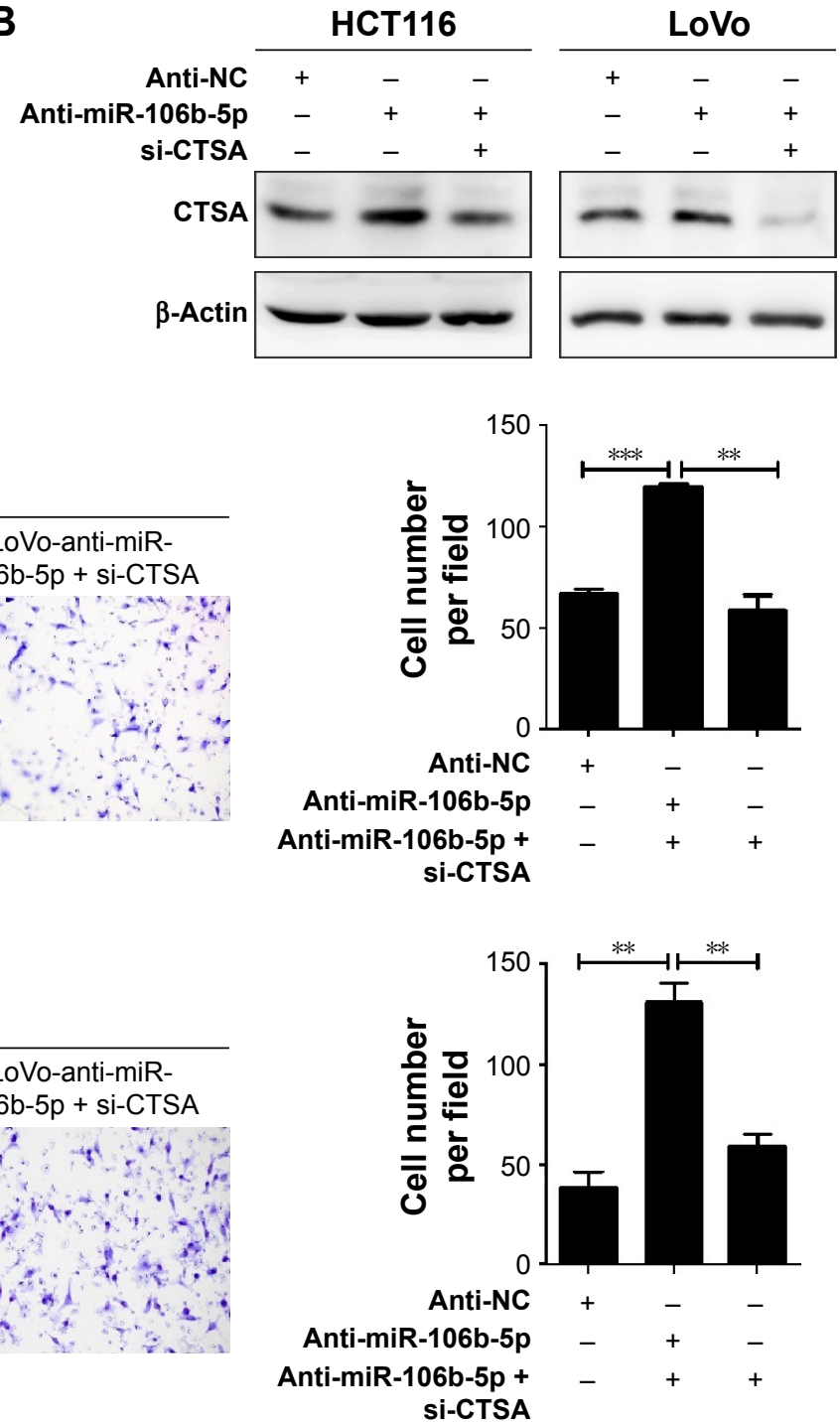

Figure 4 miR-106b-5p suppresses CRC cell migration and invasion by targeting CTSA.

Notes: (A) Silencing of CTSA in HCTII6 and LoVo cells after transfection with a specific si-CTSA was confirmed by Western blot. $\beta$-Actin served as an internal control.

(B) Western-blot analysis was used to detect CTSA expression in LoVo and HCTII6 cells transfected with an miR-106b-5p inhibitor, si-CTSA, or NC. $\beta$-Actin served as an internal control. (C) Migration and invasion assays were performed in LoVo cells transfected with an NC inhibitor, miR-106b-5p inhibitor, or si-CTSA (**P $<0.05$, $* * * P<0.001$ )

Abbreviations: CRC, colorectal cancer; CTSA, cathepsin A; NC, negative control. 
knockdown on CRC cell migration and invasion compared to that in the control group (Figure 4C). Our results indicated that miR-106b-5p inhibits CRC cell metastasis in a CTSAmediated manner. Thus, we found that miR-106b-5p functions by regulating its target CTSA in CRC.

\section{CTSA upregulation is inversely correlated with miR-106b-5p expression in CRC}

As CTSA is a direct target of miR-106b-5p, we next determined the correlation of CTSA protein expression and miR-106b-5p levels in the $78 \mathrm{CRC}$ tissues and matched nontumor tissues. Immunohistochemical staining confirmed that CTSA was significantly upregulated in CRC $(P=0.0012$; Figure 5A and B). Furthermore, Spearman's correlation analysis showed that high CTSA expression was more likely in CRCs with low levels of miR-106b-5p ( $P=0.039$; Figure 5C), and increased CTSA was associated with lymph node metastasis ( $P=0.012$; Figure 5D), suggesting that CTSA upregulation may result from miR-106b-5p repression in CRC. We also analyzed the CTSA protein expression in 49 primary CRC and liver metastases, revealing that CTSA expression was higher in the liver metastasis samples than in the primary CRC samples ( $P<0.0001$; Figure 5E), which supported the abovementioned metastasis-inducing role of CTSA in CRC cells.

Discussion miRNAs are well known to be key components of tumorigenesis, as they participate in many cellular processes, including cell proliferation, differentiation, and death. The effects of miRNAs are based on their regulation of the expression of many cancer-related genes through post-transcriptional repression. As previously reported, a panel of miRNAs was altered in CRC tissues, suggesting that variations in miRNA expression are common events in colorectal tumorigenesis. $^{21}$

miR-106b-5p is located on human chromosome 7q22.1 and belongs to the miR-106b-25 family (including miR-106b, miR-93, and miR-25), which has been reported to play vital roles in several types of cancer, including hepatocellular carcinoma, ${ }^{18}$ prostate carcinoma, ${ }^{22}$ breast carcinoma, ${ }^{23}$ gastric carcinoma, ${ }^{24}$ and laryngeal carcinoma. ${ }^{25}$ miR-106b-5p has been identified as an oncogenic miRNA in hepatocellular carcinoma, laryngeal carcinoma, breast carcinoma, and glioma. ${ }^{18,25-27}$ Other members of the miR-106b-25 family, including miR-93 and miR-25, have been reported to suppress the growth of CRC stem cells. ${ }^{28}$ However, nothing to date has been reported regarding the function of miR-106b-5p in CRC. In this study, we showed that $106 \mathrm{~b}-5 \mathrm{p}$ was frequently downregulated in human CRC and that lower miR-106b-5p expression tended to be associated with more advanced tumor-node-metastasis stages (stage I/II vs stage III/IV, $P=0.006$ ), suggesting that low miR-106b-5p expression is associated with CRC progression. Further examination showed that miR-106b-5p overexpression suppressed CRC metastasis in vitro and in vivo. Data from the current study suggest that miR-106b-5p acts as a novel metastasis suppressor in CRC and that downregulated miR-106b-5p contributes to lymph node metastasis and tumor progression in CRC patients. Therefore, we speculate that the functions of miR106b-25 family members are tissue specific.

Mature miRNAs are generated from primary precursors encoded by genes involving two subsequent RNA cleavage steps. ${ }^{29}$ When we overexpressed miR-106b-5p by lentiviral overexpression of the pre-miR-106b sequence, the intrinsic mature miR-106-3p was simultaneously overexpressed with a complementary sequence of miR-106b-5p. For that reason, overexpressing miR-106b-3p did not affect CRC cell migration and invasion. Thus, we excluded the side effect of miR-106b-3p overexpression on functional assays using cells that stably express miR-106b.

The family of cysteine cathepsins includes several members (eg, cathepsins A, B, C, F, H, K, L, O, S, V, W, and $\mathrm{X})$ that share a conserved active site. ${ }^{30,31}$ Several members of the cysteine cathepsin family have been implicated in cancer progression on the basis of their increased expression, activity, and mislocalization in various human and mouse tumors. ${ }^{32-34}$ Furthermore, their increased expression correlates with more aggressive tumors and poorer prognoses for patients. ${ }^{33,34}$ Cathepsins have been demonstrated to play roles in cancer metastasis by contributing to degradation of the basement membrane confining the tumor, thereby facilitating invasion. ${ }^{35-37}$ Among cathepsins, the levels of cysteine CTSA are increased in metastatic melanoma compared to those in primary melanoma and are associated with worse prognosis. ${ }^{20}$ These results were in accordance with our findings. Together, the observations reported in the abovementioned studies and herein demonstrate that CTSA is more highly expressed in liver metastasis than in primary $\mathrm{CRC}$ and that reducing CTSA inhibits $\mathrm{CRC}$ cell migration and invasion by involving the miR-106b-5p-modulating network. These data, for the first time, suggest that CTSA is a potential oncogene in CRC. However, the detailed mechanism by which CTSA influences CRC cell migration and invasion has not yet been elucidated. Further studies involving other cell migration and invasion genes in CRC may be performed in future. 
A

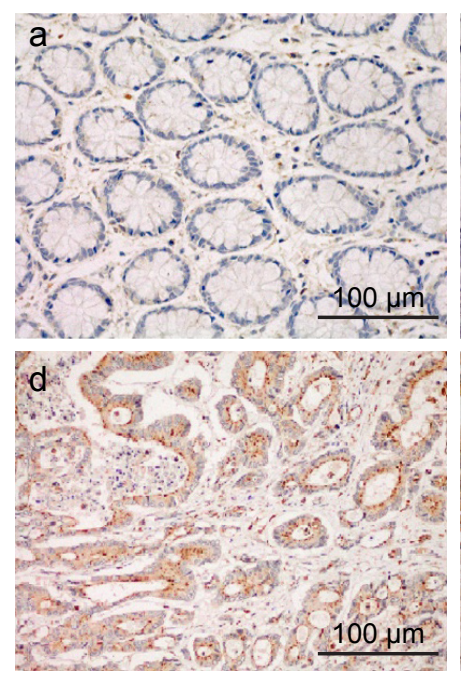

B

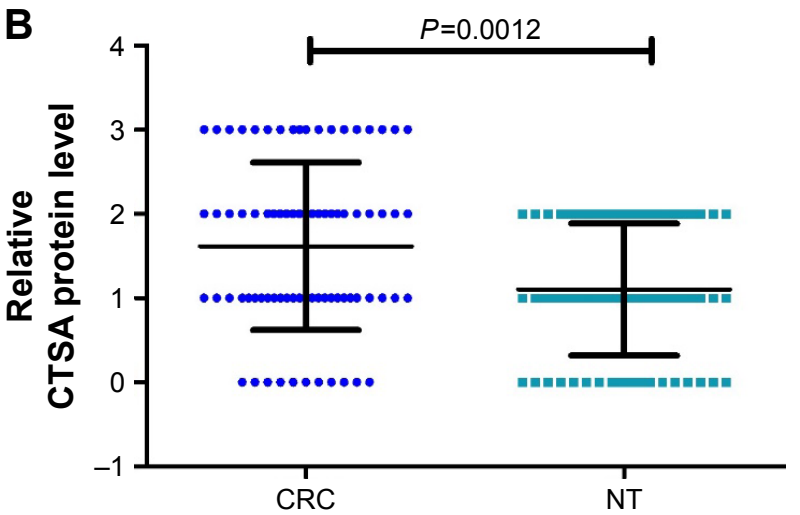

D

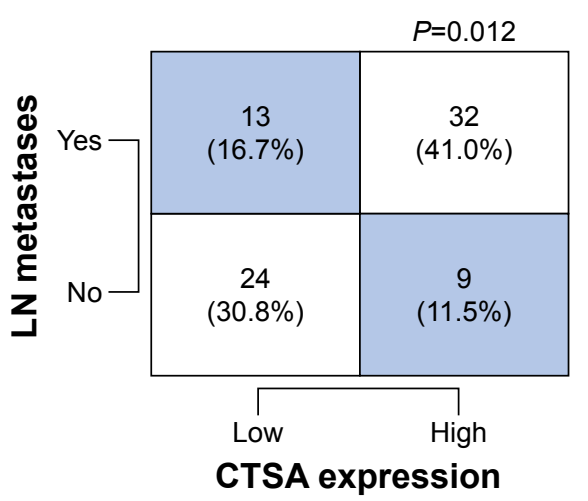

E
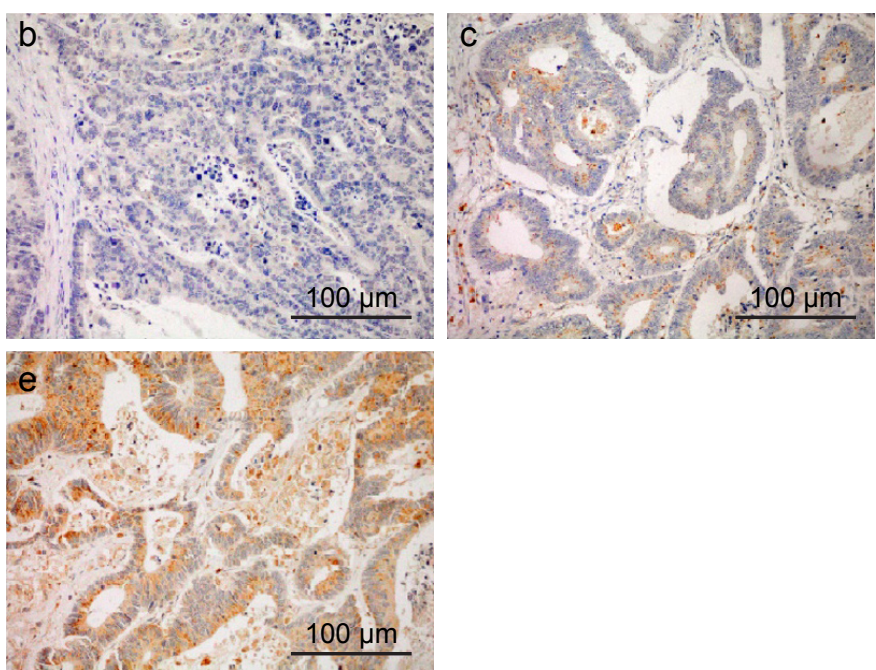
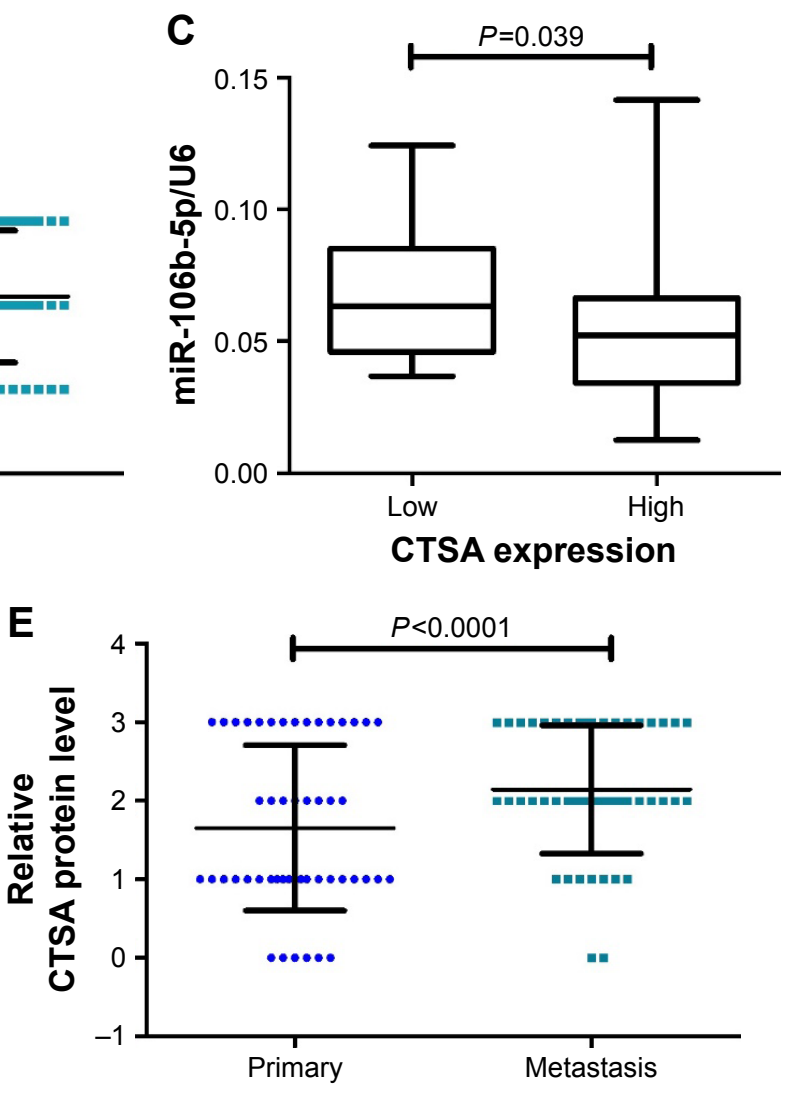

Figure 5 Upregulation of CTSA is inversely correlated with miR-106b-5p expression in CRC.

Notes: (A) Representative immunohistochemical staining of the CTSA protein in 78 CRC tissues and their corresponding NT tissues. The staining intensities are represented as follows: (a) nontumorous case I, score 0; (b) CRC case I, score 0; (c) CRC case 2, score I; (d) CRC case 3, score 2; and (e) CRC case 4, score 3. Original magnification, 200x. (B) Statistical analysis of CTSA expression according to the scoring. (C) Correlation between CTSA expression and miR-106b-5p levels in the 78 CRC tissue samples. The expression levels of CTSA were classified into low (scores of 0 and I) and high groups (scores of 2 and 3 ) according to the scores of CTSA immunohistochemical staining. (D) High expression of CTSA in CRC was associated with LN metastasis. (E) Different CTSA protein expression levels between primary CRC and liver metastases. Abbreviations: CRC, colorectal cancer; CTSA, cathepsin A; LN, lymph node; NT, nontumor.

\section{Conclusion}

Our results show that as an important antimetastatic miRNA, miR-106b-5p is downregulated and associated with lymph node metastasis in CRC. Forced expression of miR-106b-5p suppresses CRC cell invasion and metastasis by directly targeting CTSA. These findings suggest that the frequently downregulated miR-106b-5p in CRC contributes to CRC metastasis and progression and that miR-106b-5p may have therapeutic potential to suppress metastasis of CRC. 


\section{Acknowledgments}

This study was supported by the National Human Genetic Resources Sharing Service Platform (2005DKA21300), National Natural Science Foundation of China (81602078, 81472220), Shanghai Science and Technology Development Fund (17ZR1406500 and 15ZR 1407400), and Hospital Foundation of Fudan University Shanghai Cancer Center (YJ201704).

\section{Disclosure}

The authors report no conflicts of interest in this work.

\section{References}

1. Siegel RL, Miller KD, Jemal A, Statistics C. Cancer statistics, 2017. CA: A Cancer Journal for Clinicians. 2017;67(1):7-30.

2. Cunningham D, Atkin W, Lenz HJ, et al. Colorectal cancer. Lancet. 2010;375(9719):1030-1047.

3. Esquela-Kerscher A, Slack FJ. Oncomirs - microRNAs with a role in cancer. Nat Rev Cancer. 2006;6(4):259-269.

4. Zheng B, Liang L, Wang C, et al. MicroRNA-148a suppresses tumor cell invasion and metastasis by downregulating ROCK1 in gastric cancer. Clin Cancer Res. 2011;17(24):7574-7583.

5. Wang Q, Huang Z, Guo W, et al. microRNA-202-3p inhibits cell proliferation by targeting ADP-ribosylation factor-like $5 \mathrm{~A}$ in human colorectal carcinoma. Clin Cancer Res. 2014;20(5):1146-1157.

6. Deng S, Calin GA, Croce CM, Coukos G, Zhang L. Mechanisms of microRNA deregulation in human cancer. Cell Cycle. 2008;7(17): 2643-2646.

7. Li Y, Vandenboom TG, Wang Z, et al. miR-146a suppresses invasion of pancreatic cancer cells. Cancer Res. 2010;70(4):1486-1495.

8. Croce CM. Causes and consequences of microRNA dysregulation in cancer. Nat Rev Genet. 2009;10(10):704-714.

9. Medina PP, Nolde M, Slack FJ. OncomiR addiction in an in vivo model of microRNA-21-induced pre-B-cell lymphoma. Nature. 2010; 467(7311):86-90.

10. Liu J, Chen Z, Xiang J, Gu X. MicroRNA-155 acts as a tumor suppressor in colorectal cancer by targeting CTHRC1 in vitro. Oncol Lett. 2018; 15(4):5561-5568.

11. Ou C, Sun Z, Li X, et al. MiR-590-5p, a density-sensitive microRNA, inhibits tumorigenesis by targeting YAP1 in colorectal cancer. Cancer Lett. 2017;399:53-63.

12. Gong H, Fang L, Li Y, et al. miR-873 inhibits colorectal cancer cell proliferation by targeting TRAF5 and TAB1. Oncol Rep. 2018;39(3): 1090-1098.

13. Ma Y, Zhang P, Wang F, et al. Elevated oncofoetal miR-17-5p expression regulates colorectal cancer progression by repressing its target gene P130. Nat Commun. 2012;3:1291.

14. Humphreys KJ, Cobiac L, Le Leu RK, van der Hoek MB, Michael MZ. Histone deacetylase inhibition in colorectal cancer cells reveals competing roles for members of the oncogenic miR-17-92 cluster. Mol Carcinog. 2013;52(6):459-474.

15. Iseki Y, Shibutani M, Maeda K, et al. MicroRNA-96 Promotes Tumor Invasion in Colorectal Cancer via RECK. Anticancer Res. 2018;38(4):2031-2035.

16. Huang Z, Huang S, Wang Q, et al. MicroRNA-95 promotes cell proliferation and targets sorting Nexin 1 in human colorectal carcinoma. Cancer Res. 2011;71(7):2582-2589.
17. Savita U, Karunagaran D. MicroRNA-106b-25 cluster targets $\beta$-TRCP2, increases the expression of Snail and enhances cell migration and invasion in H1299 (non small cell lung cancer) cells. Biochem Biophys Res Commun. 2013;434(4):841-847.

18. Shen G, Jia H, Tai Q, Li Y, Chen D. miR-106b downregulates adenomatous polyposis coli and promotes cell proliferation in human hepatocellular carcinoma. Carcinogenesis. 2013;34(1):211-219.

19. Lu J, Wei JH, Feng ZH, et al. miR-106b-5p promotes renal cell carcinoma aggressiveness and stem-cell-like phenotype by activating Wnt/B-catenin signalling. Oncotarget. 2017;8(13):21461-21471.

20. Kozlowski L, Wojtukiewicz MZ, Ostrowska H. Cathepsin A activity in primary and metastatic human melanocytic tumors. Arch Dermatol Res. 2000;292(2-3):68-71.

21. Calin GA, Croce CM. MicroRNA signatures in human cancers. Nat Rev Cancer. 2006;6(11):857-866.

22. Hudson RS, Yi M, Esposito D, et al. MicroRNA-106b-25 cluster expression is associated with early disease recurrence and targets caspase-7 and focal adhesion in human prostate cancer. Oncogene. 2013;32(35):4139-4147.

23. Smith AL, Iwanaga R, Drasin DJ, et al. The miR-106b-25 cluster targets Smad7, activates TGF- $\beta$ signaling, and induces EMT and tumor initiating cell characteristics downstream of Six 1 in human breast cancer. Oncogene. 2012;31(50):5162-5171.

24. Kim YK, Yu J, Han TS, et al. Functional links between clustered microRNAs: suppression of cell-cycle inhibitors by microRNA clusters in gastric cancer. Nucleic Acids Res. 2009;37(5):1672-1681.

25. Cai K, Wang Y, Bao X. MiR-106b promotes cell proliferation via targeting RB in laryngeal carcinoma. $J$ Exp Clin Cancer Res. 2011;30:73.

26. Zhang A, Hao J, Wang K, et al. Down-regulation of miR-106b suppresses the growth of human glioma cells. J Neurooncol. 2013; 112(2):179-189.

27. Li N, Miao Y, Shan Y, et al. MiR-106b and miR-93 regulate cell progression by suppression of PTEN via PI3K/Akt pathway in breast cancer. Cell Death Dis. 2017;8(5):e2796.

28. Yu XF, Zou J, Bao ZJ, Dong J. miR-93 suppresses proliferation and colony formation of human colon cancer stem cells. World J Gastroenterol. 2011;17(42):4711-4717.

29. Lee YS, Nakahara K, Pham JW, et al. Distinct roles for Drosophila Dicer-1 and Dicer-2 in the siRNA/miRNA silencing pathways. Cell. 2004; 117(1):69-81.

30. Turk V, Turk B, Turk D. Lysosomal cysteine proteases: facts and opportunities. Embo J. 2001;20(17):4629-4633.

31. Mohamed MM, Sloane BF. Cysteine cathepsins: multifunctional enzymes in cancer. Nat Rev Cancer. 2006;6(10):764-775.

32. Xu X, Yuan G, Liu W, Zhang Y, Chen W. Expression of cathepsin L in nasopharyngeal carcinoma and its clinical significance. Exp Oncol. 2009;31(2):102-105.

33. Berdowska I. Cysteine proteases as disease markers. Clin Chim Acta. 2004;342(1-2):41-69.

34. Turk V, Turk B, Guncar G, Turk D, Kos J. Structure Lysosomal Cathepsins: role in antigen processing and presentation, and cancer. Adv Enzyme Regul. 2002;42:285-303.

35. Kos J, Lah TT. Cysteine proteinases and their endogenous inhibitors: target proteins for prognosis, diagnosis and therapy in cancer (review). Oncol Rep. 1998;5(6):1349-1361.

36. Roshy S, Sloane BF, Moin K. Pericellular cathepsin B and malignant progression. Cancer Metastasis Rev. 2003;22(2/3):271-286.

37. Gohring UJ, Scharl A, Thelen U, Ahr A, Crombach G, Titius BR. Prognostic value of cathepsin D in breast cancer: comparison of immunohistochemical and immunoradiometric detection methods. J Clin Pathol. 1996;49(1):57-64. 


\section{Publish your work in this journal}

OncoTargets and Therapy is an international, peer-reviewed, open access journal focusing on the pathological basis of all cancers, potential targets for therapy and treatment protocols employed to improve the management of cancer patients. The journal also focuses on the impact of management programs and new therapeutic agents and protocols on
Dovepress

patient perspectives such as quality of life, adherence and satisfaction. The manuscript management system is completely online and includes a very quick and fair peer-review system, which is all easy to use. Visit http://www.dovepress.com/testimonials.php to read real quotes from published authors.

\footnotetext{
Submit your manuscript here: http://www.dovepress.com/oncotargets-and-therapy-journal
} 